\title{
Transmitter diversity verification on ARTEMIS geostationary satellite
}

\author{
Ramon Mata Calvo**a ${ }^{\mathrm{a}}$ Peter Becker ${ }^{\mathrm{a}}$, Dirk Giggenbach ${ }^{\mathrm{a}}$, Florian Moll ${ }^{\mathrm{a}}$, \\ Malte Schwarzer ${ }^{\mathrm{b}}$, Martin Hinz ${ }^{\mathrm{b}}$, Zoran Sodnik ${ }^{\mathrm{c}}$ \\ ${ }^{a}$ Deutsches Zentrum für Luft- und Raumfahrt (DLR), Oberpfaffenhofen, 82234 Weßling, Germany; \\ ${ }^{\mathrm{b}}$ Cassidian Optronics, Oberkochen, Germany; \\ ${ }^{c}$ European Space Agency, Noordwijk, The Netherlands
}

\begin{abstract}
Optical feeder links will become the extension of the terrestrial fiber communications towards space, increasing data throughput in satellite communications by overcoming the spectrum limitations of classical RF-links. The geostationary telecommunication satellite Alphasat and the satellites forming the EDRS-system will become the next generation for high-speed data-relay services. The ESA satellite ARTEMIS, precursor for geostationary orbit (GEO) optical terminals, is still a privileged experiment platform to characterize the turbulent channel and investigate the challenges of free-space optical communication to GEO. In this framework, two measurement campaigns were conducted with the scope of verifying the benefits of transmitter diversity in the uplink. To evaluate this mitigation technique, intensity measurements were carried out at both ends of the link. The scintillation parameter is calculated and compared to theory and, additionally, the Fried Parameter is estimated by using a focus camera to monitor the turbulence strength.
\end{abstract}

Keywords: Geo Feeder links, free-space optical communications, uplink, transmitter diversity

\section{INTRODUCTION}

Optical terminals on GEO platforms are becoming a key element in future satellite communications. Satellite telecommunications must follow the increasing bandwidth requirements and overcome the spectral limitations of classical radio-frequency (RF) links. High-speed internet, television and telephone over satellite provided nowadays via Ka-Band may switch the RF Feeder-links to optical technologies, in order to reach throughputs over Tbitps.

The first dedicated European Data Relay System (EDRS) satellite is planned to be launched on 2014 and Alphasat, precursor of the EDRS System, is already in orbit. The EDRS System scope is to reduce the time delays in transmitting big amounts of data. Satellites on a GEO will relay data from and to other satellites, airplanes or ground stations. The Laser Communication Terminal (LCT) on-board EDRS satellites can provide bidirectional inter-satellite links to other satellites, but also direct optical downlinks to ground. The next ESA LEO Earth observation (EO) satellites, Sentinel 1 to 3 , will be launched during the next two years. They will use the on-board LCT to send the collected data back via the EDRS satellites [1].

Therefore, communications to GEO orbit satellites is a promising scenario for free-space optical communications, which started more than ten years ago with the ESA Semiconductor inter-satellite experiment (SILEX). Inter-satellite links were the framework of the SILEX project with two main objectives: to demonstrate the feasibility and performance of inter-satellite links and to relay video data from a LEO satellite to a ground station [2]. In 2001 there was the first image transmission from the LEO satellite SPOT-4 to the SPOTIMAGE ground station in Toulouse via the GEO Satellite ARTEMIS [3]. ARTEMIS, launched in 2001 was developed by Alenia for ESA and since November 2001 bidirectional links has been established between ARTEMIS and the ESA Optical Ground Station (OGS) on Canary Islands [4].

In this paper, we present the results of two measurement campaigns, carried out during October 2012 and April 2013. The main scope of both campaigns was to analyze the transmitter diversity mitigation effect on the uplink scintillation. Experiments were also carried out to improve the tracking performance, reducing the receiver aperture. These measurement campaigns were possible thanks to the collaboration of IAC (Instituto de Astrofísica de Canarias).

*ramon.matacalvo@dlr.de; phone +49 815328 3448; fax +49 815328 2844; www.dlr.de

Copyright 2014 Society of Photo-Optical Instrumentation Engineers. This paper was published in SPIE Photonics West 2014 and is made available as an electronic reprint with permission of SPIE. One print or electronic copy may be made for personal use only. Systematic reproduction and distribution, duplication of any material in this paper for a fee or for commercial purposes, or modification of the content of the paper are prohibited. 
This paper is focused on characterization of the delay line that is deployed to create an uncorrelated second laser beam out of one common source. It is organized as follows: section II introduces the main theoretical background, section III describes the measurement setup, section IV presents the results and section $\mathrm{V}$ discusses the main conclusions.

\section{BACKGROUND}

In this section, we will give an overview to the SILEX test-bench (i.e. the optical system setup in the ESA OGS), the laser coherence and the main theoretical parameters involved in free-space optics through the atmosphere: the structure parameter profile, the isoplanatic angle, the Fried parameter and the beam wander.

The scope of the measurements was the verification of the transmitter diversity effects using one common laser source for two-fold transmitter diversity. This is a common technique applied to reduce the scintillation at the receiver side. By transmitting $\mathrm{N}$ uncorrelated beams, the scintillation of the received signal reduces by a factor $\mathrm{N}$. Typically $\mathrm{N}$ independent sources might be used in order to obtain these $\mathrm{N}$ beams. Each beam is located at a certain distance respect to the others, to ensure that the turbulence crossed by each one is mutually uncorrelated.

In the SILEX optical bench only one laser source is available. Originally, to achieve an optical system with transmitter diversity, the source laser beam was split into four individual beams, delayed with respect to each other using three delay lines, $50 \mathrm{~mm}$ each one. Therefore, first beam was delayed $50 \mathrm{~mm}$ with respect to the second one, $100 \mathrm{~mm}$ with respect to the third and $150 \mathrm{~mm}$ with respect to the fourth. The beams were arranged in the telescope aperture forming an X, such that they do not hit the supporting spider of the secondary mirror. Nevertheless, the improvement did not reach the expected factor [5]. Therefore, another measurement campaign was proposed, improving the delay line system to ensure the independence of the transmitted beams.

\subsection{ESA Optical Ground Station (OGS) and SILEX setup}

The ESA OGS is installed in the Teide Observatory of the IAC in the Canary Islands (Spain), at a height level of $2370 \mathrm{~m}$ above the sea. The OGS is equipped with a $1 \mathrm{~m}$ diameter telescope (see left Figure 1) with Coudé Focus. The SILEX optical bench is located in the Coudé Room and it is constituted, together with the required optics and electronics, by the following main elements: the Fine-Pointing Mirror (FPM), the Acquisition Sensor (AS), the Transmit Pointing Mirror (TPM) and the Tracking Sensor (TS). The scheme is depicted in Figure 1 (right).

The laser terminal on ARTEMIS was developed based on on-off key modulation and direct detection of laser beams in the $800 \mathrm{~nm}$ range. Both terminals use wavelength discrimination to isolate the transmitted and received beams, $847 \mathrm{~nm}$ for uplink and $819 \mathrm{~nm}$ for downlink [6]. The OGS transmitter is constituted by a Ti:Sapphire laser pumped by an Argon Laser. The Ti:Sapphire laser wavelength is set at $847 \mathrm{~nm}$, for the uplink channel, with a maximum transmit power of $6 \mathrm{~W}$.
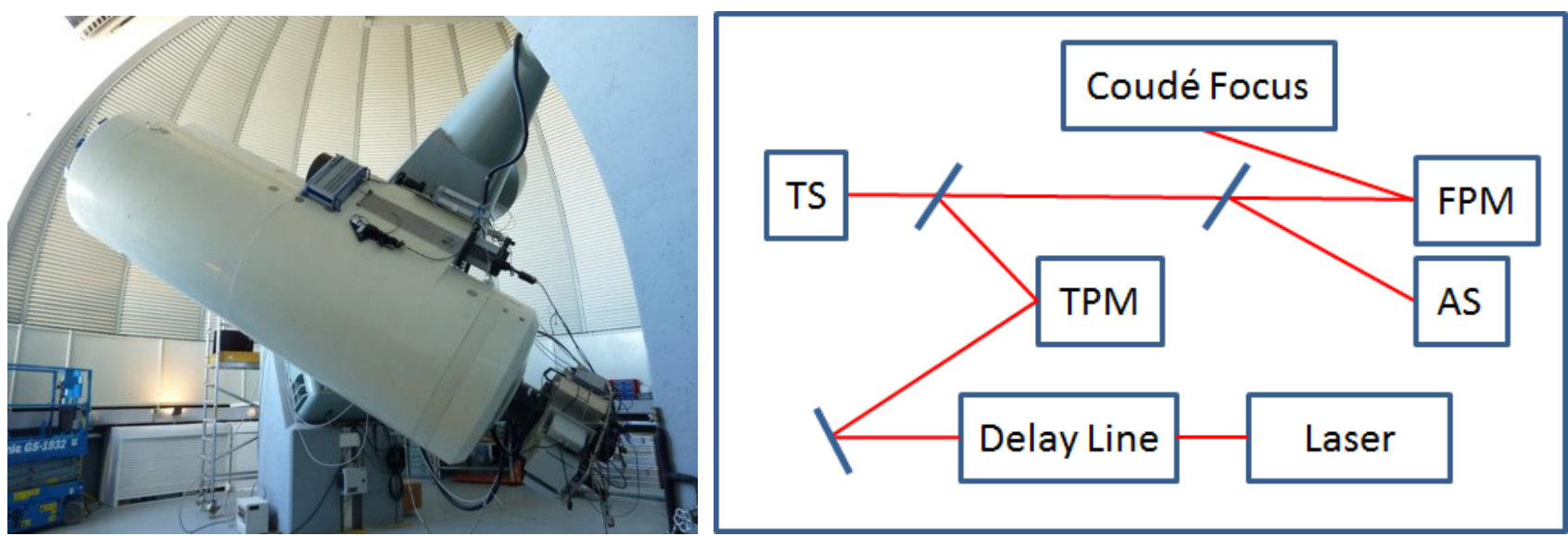

Figure 1. Telescope at the ESA Optical Ground Station (left) and SILEX setup (right)

Copyright 2014 Society of Photo-Optical Instrumentation Engineers. This paper was published in SPIE Photonics West 2014 and is made available as an electronic reprint with permission of SPIE. One print or electronic copy may be made for personal use only. Systematic reproduction and distribution, duplication of any material in this paper for a fee or for commercial purposes, or modification of the content of the paper are prohibited. 
A high power (more than 10W) laser beacon at $801 \mathrm{~nm}$ is used during the acquisition phase for the partner detection on the GEO terminal [6]; the beacon scans around $750 \mu \mathrm{rad}$ in the direction of the OGS. When the OGS AS is illuminated by the beacon, the FPM redirects the light to the TS and turns the uplink laser on. The OGS corrects for the PAA of the uplink communications beam by means of the TPM, which uses the incoming wave to close the loop. The relative motion of the GEO satellite with respect to the OGS requires the PAA correction (of around $18.5 \mu \mathrm{rad}$ ) to compensate the satellite movement during the light time-of-flight. The time needed in the line of sight correction is around 90ms [7].

The delay line splits the main beam into two independent, allowing transmitter diversity with one single laser source. After the TPM, a chromatic filter separates the uplink and downlink paths; it reflects the light coming from the TPM to the uplink channel and transmits the downlink wavelength to the TS. For alignment purposes, this filter can be substituted by a mirror that redirects the uplink wavelength to the TS and that allowed the coherence measurement of the beams.

\subsection{Laser coherence length}

The SILEX test-bench laser, Spectra-Physics 3900S, is a tunable CW Ti:Sapphire, pumped by an Argon-Ion Laser Spectra Physics Beamlock 2080. The wavelength was set to $847 \mathrm{~nm}$ for the uplink channel. The laser line-width is $0.1 \mathrm{~nm}$, which means a coherence length of $7.6 \mathrm{~mm}$. The laser line-width was verified by means of an optical spectrum analyzer.
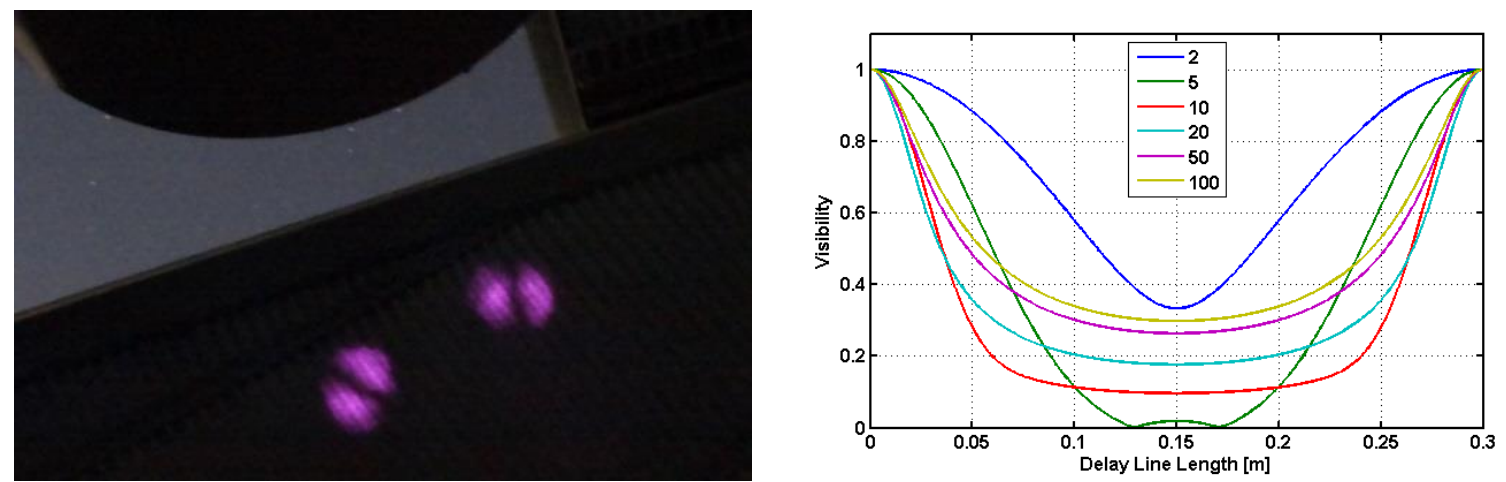

Figure 2. Transmitted beams (left) and multimode laser visibility for different numbers of longitudinal modes (right)

In Figure 2 (left) a picture of the uplink beams, reflected on the inner side of the dome cupule, is shown. A rectangular laser mode pattern can be observed, the TEM01 or TEM10; for now on we assume the TEM01. By changing the pumping laser power, the transversal mode power distribution varies slightly, but theTEM01 mode is always identifiable. The specifications of the Ti:Saphire laser states that the transmitted laser mode is TEM00, but we do not know the mode distribution of the pumping laser which may have impact on the mode of the signal laser. Unfortunately, no further information is available on this laser.

In the following we calculate the atmospheric parameters that are important for our analysis: beam wander, isoplanatic angle and Fried parameter. The fact, that the transmitted mode was not TEM00, impacts on the uplink propagation theory, i.e. a higher-order transversal mode requires a specific propagation theory for calculating the beam wander or the isoplanatic angle. Although some work exists for Hermite-Gaussian beams [8, 9], an applicable analytic expression for these parameters could not be found and therefore we will use the well-known theory based on the Gaussian beam. In the case of beam wander, it is also assumed that this effect is nearly independent from the actual mode distribution since it is mainly caused by deflection at larger eddies close to the transmitter. The results obtained by these calculations are used merely to set an order of magnitude of these parameters and are not expected to match the absolute value.

The visibility of fringes of two interfering beams of a multimode laser can be modelled as a periodic function against the optical path difference [10]. An example of this function for different numbers of longitudinal modes is displayed in Figure 2 (right). While the period of this function is determined by the cavity length of the laser, the shape depends on

Copyright 2014 Society of Photo-Optical Instrumentation Engineers. This paper was published in SPIE Photonics West 2014 and is made available as an electronic reprint with permission of SPIE. One print or electronic copy may be made for personal use only. Systematic reproduction and distribution, duplication of any material in this paper for a fee or for commercial purposes, or modification of the content of the paper are prohibited. 
the gain profile of the active medium and the spectral shape of the modes [10]. For this plot we assumed a cavity length of $15 \mathrm{~cm}$ and a Gaussian gain profile with a very narrow linewidth of the individual longitudinal modes.

\subsection{Turbulence profiles}

For the following theoretical calculations three turbulence profiles were used for the structure parameter of the index-ofrefraction turbulence: the modified Hufnagel-Valley (MHV), the Izaña Day Model (IDM) and the Izaña Night Model (INM). The last two models are empirical models derived from a measurement campaign at the OGS, which took place in 1995 [11].

The most common structure parameter profile is the Hufnagel-Valley (HV), which is valid at sea level. But the ground station is located at 2370 meters over the sea and just truncating the HV profile would lead to an underestimation of the turbulence strength. Therefore a modification is introduced to take the ground station altitude into account [12]. The expression of the MHV profile is:

$$
C_{n}^{2}(h)=0.00594(v / 27)^{2}\left(10^{-5} h\right)^{10} e^{-h / 1000}+2.7 \cdot 10^{-16} e^{-h / 500}+A_{0} e^{-H_{O G S} / 700} e^{-\left(h-H_{O G S}\right) / 100}
$$

This profile depends on the altitude above sea level $(h)$ and includes the OGS altitude ( $H_{O G S}$ ), the RMS cross wind velocity $(v)$ and a reference sea-level turbulence value $\left(A_{0}\right)$ for scaling according to day- and night-time. It is defined for $h>H_{O G S}$. A value of $10^{-13} \mathrm{~m}^{-2 / 3}$ was assumed for $A_{0}$ and $21 \mathrm{~m} / \mathrm{s}$ for $v$.

All three profiles are depicted in Figure 3 (left). The strongest turbulence conditions are represented by the IDM, followed by the INM and finally the MHV profile. The last one is introduced here as a matter of comparison with a standard formula. Furthermore, the third one provides another shape of the higher turbulence layers.

\subsection{Beam wander}

Beam wander is the beam displacement produced by large-scale turbulence structures, which appear close to the ground transmitter in a satellite uplink scenario. These structures change the beam path direction, producing a wandering of the beam around the satellite receiver. If the angular beam wander displacement is greater than the beam divergence, strong fading will occur. This analysis being located in the weak fluctuation regime, the standard deviation of the beam wander angle $\sqrt{\left\langle\alpha_{c}^{2}\right\rangle}$ is related to the wavelength $\lambda$, the Fried parameter $r_{0}$ and the transmit beam radius $W_{0}$ by the following equation [8]:

$$
\sqrt{\left\langle\alpha_{c}^{2}\right\rangle}=0.73\left(\frac{\lambda}{2 W_{0}}\right)\left(\frac{2 W_{0}}{r_{0}}\right)^{5 / 6}
$$

Figure 3 (right) shows the estimated values for the beam wander; values between $3 \mu$ rad and $14 \mu$ rad are expected at 30 degree elevation using the above mentioned profiles. Values are calculated for two beam diameters and for all three turbulence models. Although the absolute value of the beam wander does not change significantly within this two beam diameters, the impact in terms of fading is much greater for the $210 \mathrm{~mm}$ beam diameter, because its divergence is much smaller and therefore its footprint on the satellite is also smaller.

The SILEX test-bench establishes bidirectional links with ARTEMIS using the downlink incoming signal to point the uplink beam, the so-called pointing-by-tracking. If both uplink and downlink travel through the same atmosphere, by tracking the downlink signal, the beam wander should be completely precompensated. But in reality, beam wander cannot be perfectly compensated because of the point-ahead angle (PAA), as it is explained in the following lines.

Copyright 2014 Society of Photo-Optical Instrumentation Engineers. This paper was published in SPIE Photonics West 2014 and is made available as an electronic reprint with permission of SPIE. One print or electronic copy may be made for personal use only. Systematic reproduction and distribution, duplication of any material in this paper for a fee or for commercial purposes, or modification of the content of the paper are prohibited. 

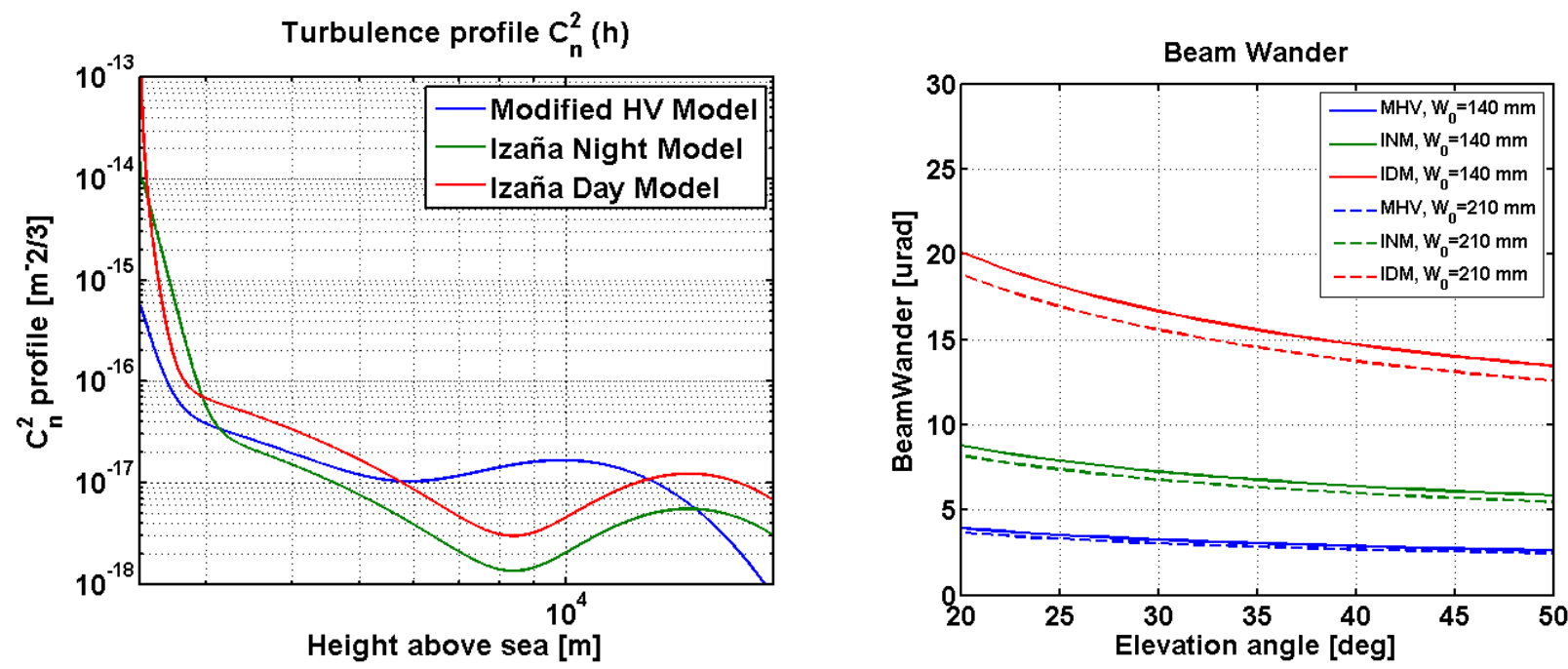

Figure 3. Turbulence profiles (left) and beam wander for a collimated TEM00 beam (right)

\subsection{Isoplanatic angle, tilt anisoplanatism and point-ahead angle}

One major issue, in satellite uplinks, is the relationship between isoplanatic angle (IPA) and point-ahead angle (PAA), when the uplink pointing uses the downlink tracking angle to also compensate the beam wander. The PAA is needed for compensating the movement of the satellite during the time needed by the light to reach it from the ground station. For a geostationary orbit the PAA is $18.5 \mu \mathrm{rad}$. The IPA is defined as the cone in which the atmospheric turbulence can be assumed constant. It is related to the index-of-refraction structure parameter profile $C_{n}^{2}$, the wave-number $k$, the height $h$ and the zenith angle $\varsigma$ by the following equation [8].

$$
\theta_{0}=\left[2.914 k^{2} \sec ^{8 / 3}(\varsigma) \int_{H_{O G S}}^{\infty} C_{n}^{2}(h) h^{5 / 3} d h\right]^{-3 / 5}
$$

The weighting factor inside the integral leads to a strong dependence of the IPA on the high-altitude turbulence. If uplink and downlink travel through the same atmosphere, the angle-of-arrival fluctuations of the downlink beam could be used to pre-correct the uplink beam wander (also assuming same beam size). Due to the PAA, uplink and downlink will not cross the same atmosphere. However pointing-by-tracking will be beneficial if the turbulence between uplink and downlink is still correlated to a certain degree.

In Figure 4 (left), the PAA is compared to the IPA. For all turbulence profiles and elevation angles between 20 and 50 degrees, the PAA is larger than the IPA. This difference does not imply a complete decorrelation between uplink and downlink; actually pointing by tracking is beneficial in communicating with ARTEMIS as demonstrated by the past measurement campaigns. But significant extra fading due to pointing errors are to be expected, on top of the scintillation due to the small-scale turbulence structures.

Indeed the IPA is exploited in many adaptive optics systems and takes into account the whole phase distortion, which includes not only the tilt but also higher order distortions. In [13], the tilt anisoplanatism is derived by applying a Zernike filter function:

$$
\theta_{T A}=\frac{0.184 \lambda D^{1 / 6}}{\left(\sec ^{3}(\xi) \int_{H_{O G S}}^{H_{A R T}} C_{n}^{2}(h) h^{2} d h\right)^{1 / 2}}
$$

Copyright 2014 Society of Photo-Optical Instrumentation Engineers. This paper was published in SPIE Photonics West 2014 and is made available as an electronic reprint with permission of SPIE. One print or electronic copy may be made for personal use only. Systematic reproduction and distribution, duplication of any material in this paper for a fee or for commercial purposes, or modification of the content of the paper are prohibited. 

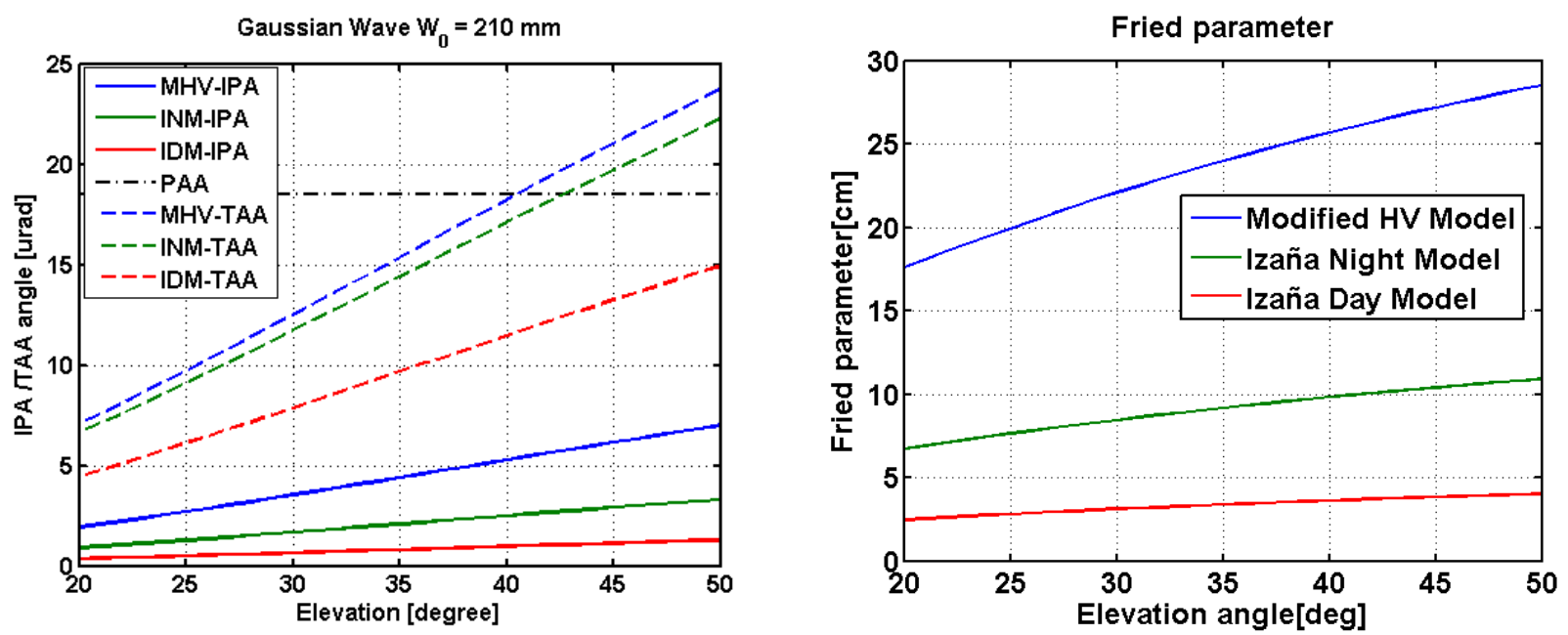

Figure 4. IPA and TAA versus PAA (left) and Fried parameter (right)

The tilt anisoplanatism angle (TAA) $\theta_{T A}$ represents the angle between two sources, at which one of them jitters with a magnitude of half of its diffraction-limited divergence, with respect to the other one. Since we are performing pointing by tracking, we assume here that the angle at which the uplink pointing jitter is half of the diffraction limited beam divergence is represented by $\theta_{T A}$. Although, this equation does not take the TEM01 mode into account and assumes some approximations that are not fully accomplished, we use the calculated value to estimate the TAA order of magnitude.

In Figure 4 (left), we plot the TAA together with the above commented IPA. At 20 degrees elevation, the TAA is around $12 \mu \mathrm{rad}$ for the MHV and INM profiles, for the IDM profile the TAA decreases at around $7 \mu$ rad. These values are closer to the PAA than the IPA, but they are still smaller, therefore deep fades are expected due to pointing errors.

\subsection{Fried parameter}

The Fried parameter $r_{0}$ is a measure for the transversal beam coherence and it depends on the path-integrated turbulence strength. It is defined as follows [8]:

$$
r_{0}=\left[0.42 \cdot k^{2} \cdot \sec (\varsigma) \int_{H_{O G S}}^{\infty} C_{n}^{2}(h) d h\right]^{-3 / 5}
$$

The Fried parameter is related to the index-of-refraction structure parameter profile $C_{n}^{2}$, the wave-number $k$, the height $h$ and the zenith angle $\varsigma$.

The Fried parameter is represented between $20^{\circ}$ and $50^{\circ}$ elevation angle in Figure 4 (right). The three models provide a wide range of values between $4 \mathrm{~cm}$ and $22 \mathrm{~cm}$ at $30^{\circ}$ elevation.

Fried parameter can be related with the long term focus spot images. At the focus plane of the telescope, the beam forms speckles that change with the time due to the atmospheric turbulence. Overlapping several focus images, constituted by such speckles, one can get a mean focus spot image. By increasing the number of averaging samples, this mean spot tends to a Gaussian shape. The width of this average shape is related to the Fried parameter as explained in [14].

Copyright 2014 Society of Photo-Optical Instrumentation Engineers. This paper was published in SPIE Photonics West 2014 and is made available as an electronic reprint with permission of SPIE. One print or electronic copy may be made for personal use only. Systematic reproduction and distribution, duplication of any material in this paper for a fee or for commercial purposes, or modification of the content of the paper are prohibited. 


\section{MEASUREMENT SETUP}

Two major modifications were introduced in the SILEX optical test-bench: a new delay line system and a turbulence characterization setup.

\subsection{Delay Line configuration}

In these experiments, we opted for using only two beams, in order to reduce the hardware complexity. A new beam delay system (BDS) was designed and built by Cassidian Optronics, with larger delays, to ensure two incoherent laser beams.

The BDS is an optical delay system with two operational modes. The first one is a non-splitting mode were an entrance beam goes directly through the system. Incoming and outgoing beam have the same intensity but the polarization is rotated from vertical to horizontal. The second is a splitting mode where an entrance beam is split into two single beams with an intensity of $50 \%$ each. Furthermore these two beams can temporary be shifted to each other by an optical delay line. Both channels are designed to ensure the correct polarization for the subsequent optical system and withstand the input laser power of 10W @ 847nm.

The BDS is designed as an electrical powered mechanics which provides the possibility to create one or two output beams with an adjustable temporal delay by using a notebook or controller. This enables the user to select an operational mode and change delay length without opening the device and losing the link to Artemis. Furthermore the laser safety (10W@847nm) is not affected because all protective covers are in their nominal position.

Figure 5 shows the BDS as CAD design status and as the final integrated mechanism including all optical and mechanical components as implemented into the optical bench on Tenerife.
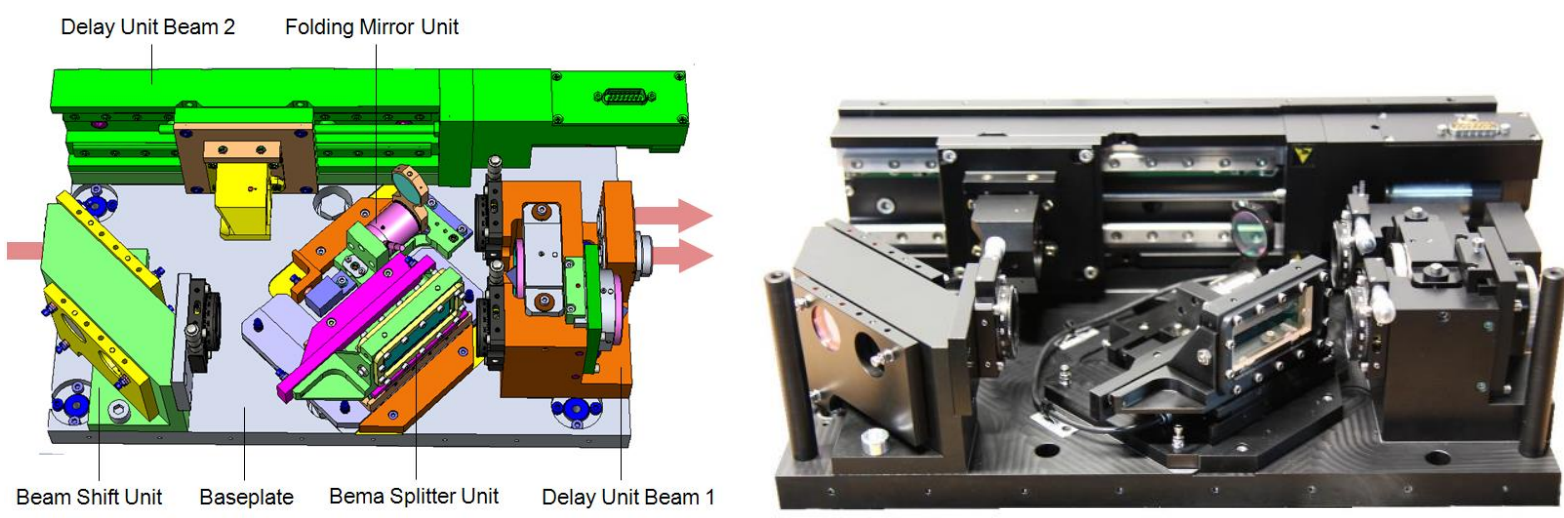

Figure 5. CAD Model of the BDS with subunits (left) and final BDS front view (right)

The delay line of the BDS comprises two parts. First a static delay of beam 1 to compensate the lateral beam offset and to ensure a delay of $0 \mathrm{~ns}$. Second a moveable delay line into beam 2 to increase the delay between both beams up to $1 \mathrm{~ns}$ (equal to $300 \mathrm{~mm}$ optical length). This adjustment from 0 to $300 \mathrm{~mm}$ is realized by a beam reflectance element (corner cube) and a motorized rail stage with encoder for calculating the delay length.

For operation of the BDS a Controller Unit was built up which includes the supply and communication interface of the three electric moveable stages. To calibrate the pointing of the two outputs within a tolerance of 1 arcsec during operation of the BDS a measurement system consisting of a collimator and attenuators was integrated to the optical bench. By shutting the beams after each other the position onto the camera can be selected. Due to that a pointing misalignment between both beams will lead to a spot shift at the camera and can be readjusted by moving the tip-tilt stage till no tilt can be detected.

The main requirements were the pointing stability as well as parallelism of beam 1 and beam 2 during operation and movement of the optical subunits. This was calculated and specified with $<5.4 \mu \mathrm{rad}$.

Copyright 2014 Society of Photo-Optical Instrumentation Engineers. This paper was published in SPIE Photonics West 2014 and is made available as an electronic reprint with permission of SPIE. One print or electronic copy may be made for personal use only. Systematic reproduction and distribution, duplication of any material in this paper for a fee or for commercial purposes, or modification of the content of the paper are prohibited. 
To realize a pointing accuracy $\sim 1$ " in each operating modus a continuously measurement was necessary. Therefore a collimator system with integrated camera was placed onto the optical bench to measure the pointing of both beams near the exit to the telescope. This enables detection of possible misalignment independent if it is caused by BDS subunit movement or optical effects of other components on the optical bench with an accuracy of $<1$ arcsec in real time and to adjust the output beam pointing by moving the tip tilt folding mirror with a stage accuracy of 0.2 ".

\subsection{Turbulence characterization setup}

The measurement setup for the turbulence characterization was located after a beam splitter, which provides the $70 \%$ of the available incoming power. The whole power that reaches the beam splitter is $3.3 \mathrm{nW}, 1.1 \mathrm{nW}$ are used by the TS to keep the link stable. The remaining $2.2 \mathrm{nW}$ are used for a focus camera. The scheme of the main measurement components is drawn in Figure 6 (left).

The focus camera is a QImaging QICAM 1394, with $13.7 \mu \mathrm{m}$ pixel size, 12 bits and frame-rate of 20fps. The light is focused on the sensor area by means of a $1 \mathrm{~m}$ focal length doublet with AR coating and two silver coated mirrors. The recorded images are transferred by a Firewire interface to the computer. A photograph of the measurement hardware is shown in Figure 6 (right). The compression factor of the optical system before the focusing lens is 29.4; therefore, the effective focal length is 29.4 meters. With such an optical configuration the diffraction limited focal spot's Airy pattern would have a FWHM of $D_{\text {focus }}=1.024 \lambda f / D=25 \mu \mathrm{m}$ or 1.8 pixels. The atmospherically distorted wave-front creates a speckle pattern much larger, thereby enabling an estimation of $r_{0}$.

The tracking sensor is constituted by an array of 16x16 pixels. The four central pixels form a 4 quadrant detector (4QD), which provides a received power measurement with $1 \mathrm{kHz}$ sampling rate. This information together with the pointing error is preprocessed by the SILEX system and provided afterwards in an ASCII file format. The TS drives the tilt mirror with two analog signals, to keep the spot in the middle of the 4QD. The analog signal was sampled using a Picoscope 4424 with $10 \mathrm{kHz}$ sampling rate.
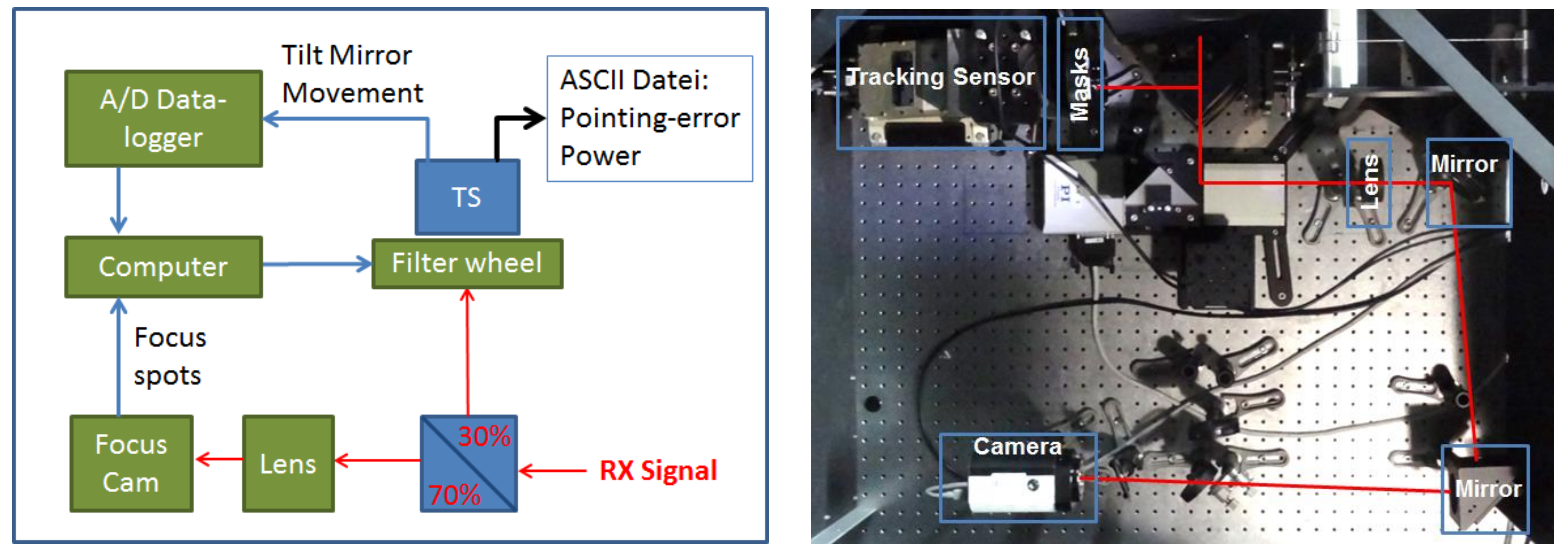

Figure 6. Measurement system scheme (left) and setup (right)

Finally a filter wheel (Starlight SXUFW-2S) was located in front of the tracking sensor to test the effect of reducing the tracking area by applying several circular aperture masks. On the wheel instead of filters, up to five different masks were mounted. Each mask contained one or two small circular holes. Masks with holes aligned to the transmitted uplink beam were tested, to proof if the tracking quality improves when pointing and tracking path are correlated. The uplink scintillation was measured and compared using the whole receiving aperture (without mask), a mask with a coincident aperture to the uplink beam and a mask with a non-coincident aperture to the uplink beam.

Copyright 2014 Society of Photo-Optical Instrumentation Engineers. This paper was published in SPIE Photonics West 2014 and is made available as an electronic reprint with permission of SPIE. One print or electronic copy may be made for personal use only. Systematic reproduction and distribution, duplication of any material in this paper for a fee or for commercial purposes, or modification of the content of the paper are prohibited. 


\section{MEASUREMENTS AND RESULTS}

In this section, we present the coherence measurements of the uplink laser, the atmospheric characterization through the Fried parameter, the uplink fading measurements and the influence of the offset between receiver and transmitter aperture location. The bidirectional link was characterized for three uplink beam FWHM divergences $4.7 \mu \mathrm{rad}(210 \mathrm{~mm}$ beam diameter) $7.1 \mu \mathrm{rad}(140 \mathrm{~mm}$ beam diameter) and $10.0 \mu \mathrm{rad}(100 \mathrm{~mm}$ beam diameter). Measurements were performed at several times. The transmitter diversity was verified with different delay values between $0 \mathrm{~mm}$ (fully correlated beams) and $300 \mathrm{~mm}$. The elevation angle was around 30 degree.

In Table 1, we have an overview of all the measurements with valid data. The day when the measurement was taken is written in the corresponding cell, with the day date followed with a capital letter: $A$ for April and $O$ for October. DLine stands for a set of delay line measurements and Masks stands for a set of measurements when a particular mask was applied. The DLine set comprises measurements with $50 \mathrm{~mm}$ delay steps between 0 and $300 \mathrm{~mm}$ and the Masks set comprises several mask configurations. Thus each of measurement sets contain between 5 and 8 single measurements.

Table 1. Measurements containing valid data for the October 2012 and April 2013 campaigns: 100, 140 and $210 \mathrm{~mm}$ denote the beam size and the leading number stands for the day date, A/O for April/October.

\begin{tabular}{|c|c|c|c|c|c|c|c|c|c|}
\hline Hour UTC & Oh & $1 \mathrm{~h}$ & $2 \mathrm{~h}$ & $4 h$ & $5 h$ & $20 \mathrm{~h}$ & $21 \mathrm{~h}$ & $22 \mathrm{~h}$ & 23h \\
\hline \multicolumn{10}{|l|}{$100 \mathrm{~mm}$} \\
\hline DLine & & & & & 270 & & 260 & & \\
\hline Masks & & & 270 & 270 & 270 & & & & \\
\hline \multicolumn{10}{|l|}{$140 \mathrm{~mm}$} \\
\hline DLine & & & & & & & $25 A, 26 A$ & & 26A \\
\hline Masks & 26A & & & & & 26A & & 25A & $25 \mathrm{~A}$ \\
\hline \multicolumn{10}{|l|}{$210 \mathrm{~mm}$} \\
\hline DLine & & 19A & & & & & 17A,22A & 17A,19A,22A & $20 A$ \\
\hline Masks & 20A & & & & & & & & 19A,20A \\
\hline
\end{tabular}

\subsection{Laser Beams Coherence}

The coherence of both beams was tested using an interferometer-like configuration. Both beams were overlapped and the strength of the interference pattern was measured through the visibility. In Figure 7 (left), the interferometer setup is shown. After the delay line, both uplink beams were attenuated and redirected to the measurement setup. By means of a mirror and a splitter, both beams were overlapped onto the camera sensor, producing the interference pattern.
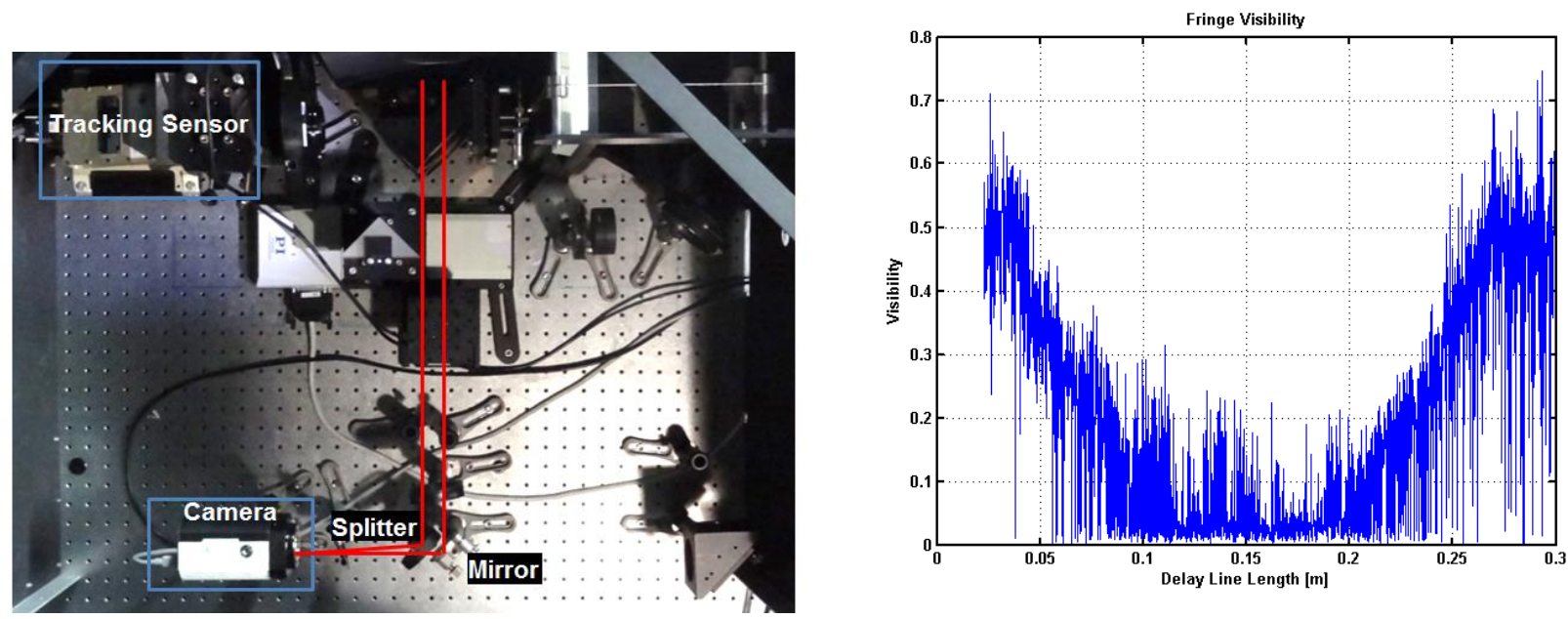

Figure 7. Interferometer setup (left) and visibility curve versus delay length (right)

Copyright 2014 Society of Photo-Optical Instrumentation Engineers. This paper was published in SPIE Photonics West 2014 and is made available as an electronic reprint with permission of SPIE. One print or electronic copy may be made for personal use only. Systematic reproduction and distribution, duplication of any material in this paper for a fee or for commercial purposes, or modification of the content of the paper are prohibited. 
The interference pattern visibility is a common measurement of the coherence quality. Ideally if two perfectly coherent beams overlap, a maximum visibility of unity is achieved. On the contrary, if the two beams are completely incoherent visibility is zero. The mathematical expression of the visibility depends on the intensity levels of the bright lines $\left(I_{2}\right)$ and the intensity level of the dark lines $\left(I_{1}\right)$ and it is as follows [15]:

$$
V=\frac{I_{2}-I_{1}}{I_{2}+I_{1}}
$$

The measurement was performed shifting continuously the delay line between 20 and $300 \mathrm{~mm}$. The calculated visibility is plot in Figure 7 (right). The visibility drops at around $100 \mathrm{~mm}$ and increases again after $200 \mathrm{~mm}$. The scope of the measurement was to validate the trend of the laser coherence with the delay line. This measurement was performed in a highly noisy environment and therefore the absolute value of the results is influenced by vibrations. However the general trend of the beam coherence with the delay line can be clearly observed.

\subsection{Turbulence strength measurement}

The Fried parameter is commonly used to characterize the strength of the turbulence influence on the beam propagation and it is closely related to the seeing parameter. The seeing is also widely used, mainly by the astronomers, and it gives also information on the turbulence strength; the best angular resolution that a telescope can achieve due to the turbulence. Near the ESA OGS in Tenerife, the IAC monitors the seeing with an automatic DIMM (Differential Image Motion Monitor). These measurements were however not synchronized with our experiments. Therefore we got two information sources of the atmospheric turbulence during the measurement campaign.

Seeing values are provided in arcseconds at zenith but from them the Fried parameter can be easily calculated at $30^{\circ}$ elevation. Both sources provided the same range of values of $r_{0}$, between 5 and $25 \mathrm{~cm}$, calculated at $30^{\circ}$ elevation. Some seeing values achieved the $35 \mathrm{~cm}$, but no measurements took place at that time. However, the seeing measurements and the Fried parameter derived from the focus images agree as it is shown in Figure 8 (left). The values derived from the DIMM and focus camera are all in reasonable order but cannot perfectly match since the observed atmospheric paths are different.
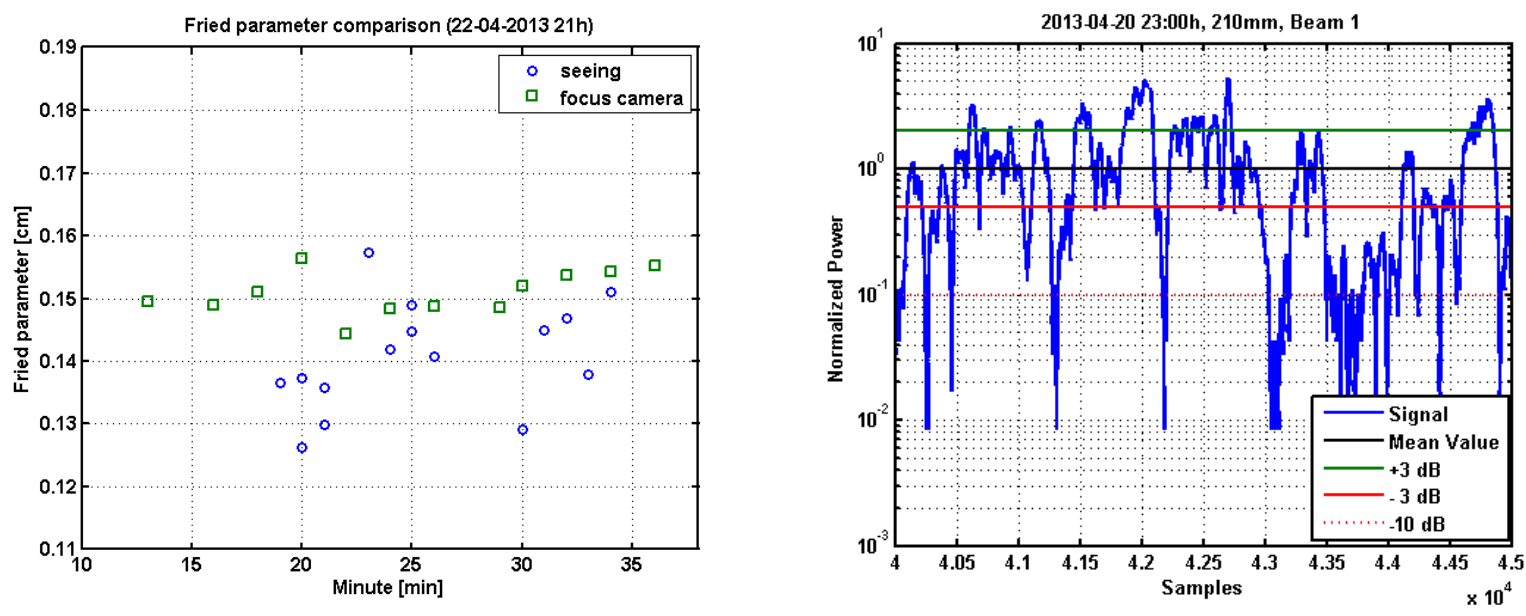

Figure 8. Fried parameter (left) and uplink power vector (right)

Copyright 2014 Society of Photo-Optical Instrumentation Engineers. This paper was published in SPIE Photonics West 2014 and is made available as an electronic reprint with permission of SPIE. One print or electronic copy may be made for personal use only. Systematic reproduction and distribution, duplication of any material in this paper for a fee or for commercial purposes, or modification of the content of the paper are prohibited. 


\subsection{Uplink channel fading}

The uplink power was measured by ARTEMIS and send back via Telemetry. An example of the measured power is plot in Figure 8 (right). Around the mean value, the received power fluctuates, having several $3 \mathrm{~dB}$ surges, $3 \mathrm{~dB}$ fades and 10 $\mathrm{dB}$ fades. This particular measurement was performed on $20^{\text {th }}$ April at $23 \mathrm{~h}$ UTC, using one uplink beam with $210 \mathrm{~mm}$ diameter. Deep fades of about $20 \mathrm{~dB}$ can also be observed and we attribute them to pointing errors. Because the IPA and the TAA are expected to be smaller than the PAA, the downlink tracking will be not able to completely compensate the beam wander. We assume that the uncorrected beam wander is responsible of these deep fades. Assuming a Gaussian distribution of the far field beam, if the separation between uplink and downlink would be equal to the TAA, a jitter on the order of half diffraction limited divergence would be expected, i.e. in the order of $1 / e^{2}$ of the intensity, let's say 10 $\mathrm{dB}$ below the on-axis value. Being the PAA larger than the TAA, deeper fades are expected. Indeed, for $10 \log _{10}\left\{\exp \left[-2\left(\theta_{P A A} / \theta_{T A A}\right)^{2}\right]\right\}$, the estimated jitter is in the order of $20 \mathrm{~dB}$. As already mentioned these calculations are used only in order to get a feeling on the order of magnitude and the calculated values are not to be taken as absolute values.

Table 2. Uplink fade duration and scintillation for two beam divergences

\begin{tabular}{|c|c|c|c|c|}
\hline & \multicolumn{2}{|c|}{$210 \mathrm{~mm}$ beam diameter } & \multicolumn{2}{|c|}{$140 \mathrm{~mm}$ beam diameter } \\
\hline Fade/Surge Time & 1 Uplink Beam & Transmitter Diversity & 1 Uplink Beam & Transmitter Diversity \\
\hline Mean -3dB [ms] & 8.2 & 2.7 & 10.6 & 3.2 \\
\hline \#Fades $-3 \mathrm{~dB} / \mathrm{s}\left[\mathrm{s}^{-1}\right]$ & 45 & 101 & 29 & 59 \\
\hline Mean -10dB [ms] & 2.7 & 1.4 & 3.9 & 1.4 \\
\hline \#Fades $-10 \mathrm{~dB} / \mathrm{s}^{2}\left[\mathrm{~s}^{-1}\right]$ & 41 & 18 & 10 & 1.5 \\
\hline Mean $+3 \mathrm{~dB}[\mathrm{~ms}]$ & 5 & 10 & 7.2 & 1.85 \\
\hline$\#$ Surges $+3 \mathrm{~dB}\left[\mathrm{~s}^{-1}\right]$ & 29 & 92 & 15.5 & 35.5 \\
\hline Scintillation [] & 1.04 & 0.46 & 0.50 & 0.18 \\
\hline Fried parameter $[\mathrm{cm}]$ & & $7 \mathrm{~cm}$ & & 9 \\
\hline
\end{tabular}

In Table 2, we summarized the mean fade duration and the fade frequency for several configurations and fade depth. These measurements were taken on $20^{\text {th }}$ and $25^{\text {th }}$ April at $23 \mathrm{~h}$. Using only one beam, the fade duration slightly increases when the beam divergence increases; i.e. when the beam diameter decreases. For larger beam diameters, the divergence is smaller and the beam wander impact is higher. That can be confirmed looking at the fade and surge frequencies: the number of fades or surges per second reduces when the beam diameter decreases. Transmitter diversity reduces the fade depth; the number of $3 \mathrm{~dB}$ fades increases and the fade duration decreases. As a matter of example, using one uplink beam of $210 \mathrm{~mm}$ diameter, the fade duration at $-10 \mathrm{~dB}$ is $2.7 \mathrm{~ms}$ with 41 fades per second. Using transmitter diversity the fade frequency and the fade duration reduces to the half of them. This reduction is more drastic for a $140 \mathrm{~mm}$ beam diameter; the number of fades per second is reduced by a factor of 6.6 and the fade time by a factor of 2.7.

The cumulative density function (CDF) of the fades duration using transmitter diversity is plot in Figure 9 (left). Both transmitter beam divergences are taken into account.

Transmitting only one uplink beam, the duration of $3 \mathrm{~dB}$ fades is below $1 \mathrm{~ms}$, the $30 \%$ of times for $140 \mathrm{~mm}$ beam diameter and around the $15 \%$ of times for $210 \mathrm{~mm}$ beam diameter. Deeper fades $(10 \mathrm{~dB})$ are often shorter; $35 \%$ of times for $140 \mathrm{~mm}$ and $25 \%$ of times for $210 \mathrm{~mm}$. With transmitter diversity (left Figure 9), the fades become shorter in time, around $65 \%$ of time fades are below $1 \mathrm{~ms}$ and $95 \%$ of time fades are below $10 \mathrm{~ms}$. 10dB fades remain still shorter in time than $3 \mathrm{~dB}$. Around $60 \%$ of the $3 \mathrm{~dB}$ fades are below $1 \mathrm{~ms}$ and $90 \%$ below $10 \mathrm{~ms}$ (140 mm beam). The $10 \mathrm{~dB}$ fades are ever shorter, i.e. $65 \%$ are below $1 \mathrm{~ms}$ and around $99 \%$ below $10 \mathrm{~ms}$.

The probability density function (PDF) is estimated using a normal kernel function. The PDFs for two beam diameters $210 \mathrm{~mm}$ and $140 \mathrm{~mm}$ are plot in Figure 9 (right). We included two delay values $150 \mathrm{~mm}$ and $300 \mathrm{~mm}$. These measurements were taken also on $20^{\text {th }}$ and $25^{\text {th }}$ April at $23 \mathrm{~h}$. The divergence impact on the received power can be observed clearly in the PDF shapes. For smaller divergences $(210 \mathrm{~mm}$, solid lines), the PDF shows higher probability

Copyright 2014 Society of Photo-Optical Instrumentation Engineers. This paper was published in SPIE Photonics West 2014 and is made available as an electronic reprint with permission of SPIE. One print or electronic copy may be made for personal use only. Systematic reproduction and distribution, duplication of any material in this paper for a fee or for commercial purposes, or modification of the content of the paper are prohibited. 
values for power levels closer to zero than for larger divergences (140mm, dashed lines). The black line represents the PDF for $0 \mathrm{~mm}$ delay, i.e. for two coherent beams, the red line for $150 \mathrm{~mm}$ delay and the blue line for $300 \mathrm{~mm}$ delay.
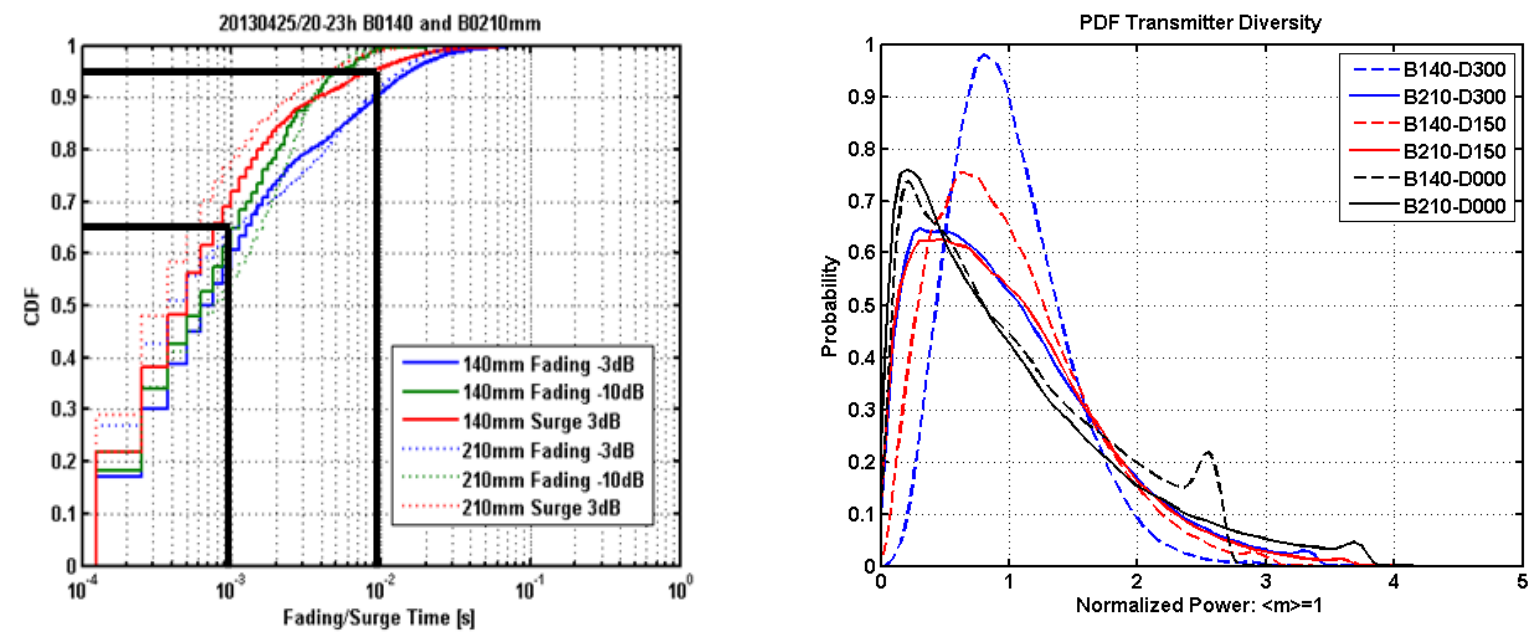

Figure 9. CDF of the fading duration with transmitter diversity (left) and estimated PDF of the uplink received power with transmitter diversity (right)

As expected, the shape approaches to an exponential function, with higher probability for values near zero (B210-D000). Some sensor saturation can be observed in the PDF for $140 \mathrm{~mm}$ diameter and $0 \mathrm{~mm}$ delay line (the small peak at 2.6 ). Saturation was quite difficult to avoid in scenarios with high power fluctuations, because of the limited dynamic range of the ARTEMIS sensor [5]. The scintillations values for these configurations are collected in Table 3 and can be graphically seen in Figure 10 (left): the blue, cyan and green lines for the $140 \mathrm{~mm}$ beam and the red and magenta lines for the $210 \mathrm{~mm}$ beam diameter.

Table 3. Scintillation values for several beam and delay configurations

\begin{tabular}{|c|c|c|c|c|c|c|}
\hline Beam-Delay & B140-D300 & B210-D300 & B140-D150 & B210-D150 & B140-D000 & B210-D000 \\
\hline Scintillation & 0.2 & 0.48 & 0.3 & 0.47 & 0.5 & 0.69 \\
\hline
\end{tabular}

From the uplink power vectors, we calculated the scintillation index (power variance normalized by the square of the mean value). The values for 1 single beam and with transmitter diversity are included in the Table 2, together with the measured Fried parameter values. In Figure 10 (left), we plot the scintillation index for transmitter diversity over the delay line length. As expected, the scintillation decreases with delay length. The higher values are obtained at delay 0 $\mathrm{mm}$, then after $50 \mathrm{~mm}$ the scintillation changes slope and in some cases, the scintillation increases after $150 \mathrm{~mm}$. The reason here is the reoccurring correlation between the two beams as shown in Figure 7 (left) with the fringe visibility.

\subsection{Offset between receiver and transmitter aperture location}

The uplink beam has a smaller diameter than the whole telescope aperture. We did experiments with $100 \mathrm{~mm}, 140 \mathrm{~mm}$ and $210 \mathrm{~mm}$ uplink beam diameters and the telescope has a $1 \mathrm{~m}$ aperture diameter. The tracking system actually uses the whole $1 \mathrm{~m}$ telescope aperture to track the downlink beam and in return, to point the uplink beam. With this experiment we wanted to test if using a smaller aperture diameter aligned with the uplink beam improves the pointing performance, i.e. if the uplink scintillation can be reduced. For that purpose, we build a set of masks that we placed in front of the TS, mounted in a filter wheel, which was controlled by the computer.

The following code was used to identify the masks: W (whole aperture), C2 (mask correlated with beam \#2) and C1 (mask correlated with beam \#1). Beams were identified with indices 1 and 2. In Figure 10 (right), the scintillation and

Copyright 2014 Society of Photo-Optical Instrumentation Engineers. This paper was published in SPIE Photonics West 2014 and is made available as an electronic reprint with permission of SPIE. One print or electronic copy may be made for personal use only. Systematic reproduction and distribution, duplication of any material in this paper for a fee or for commercial purposes, or modification of the content of the paper are prohibited. 
the mean power for several mask configurations are plot. This measurement was performed on 27 October 2012, around 5:00 AM. In this measurement we used only one beam, the beam number 2. Therefore mask $\mathrm{C} 2$ blocks received light from the whole aperture, except an aperture of $300 \mathrm{~mm}$, which is centered at the same point where beam 2 is sent in uplink, thus mask $\mathrm{C} 2$ is correlated with beam 2. Mask $\mathrm{C} 1$ is centered where the beam 1 would be transmitted, thus it is masking an uncorrelated area of the telescope versus the transmit beam 2.

The following code was used to identify the masks: W (whole aperture), C2 (mask correlated with beam \#2) and C1 (mask correlated with beam \#1). Beams were identified with indices 1 and 2. In Figure 10 (right), the scintillation and the mean power for several mask configurations are plot. This measurement was performed on 27 October 2012, around 5:00 AM. In this measurement we used only one beam, the beam number 2. Therefore mask $\mathrm{C} 2$ blocks received light from the whole aperture, except an aperture of $300 \mathrm{~mm}$, which is centered at the same point where beam 2 is sent in uplink, thus mask $\mathrm{C} 2$ is correlated with beam 2. Mask $\mathrm{C} 1$ is centered where the beam 1 would be transmitted, thus it is masking an uncorrelated area of the telescope versus the transmit beam 2.
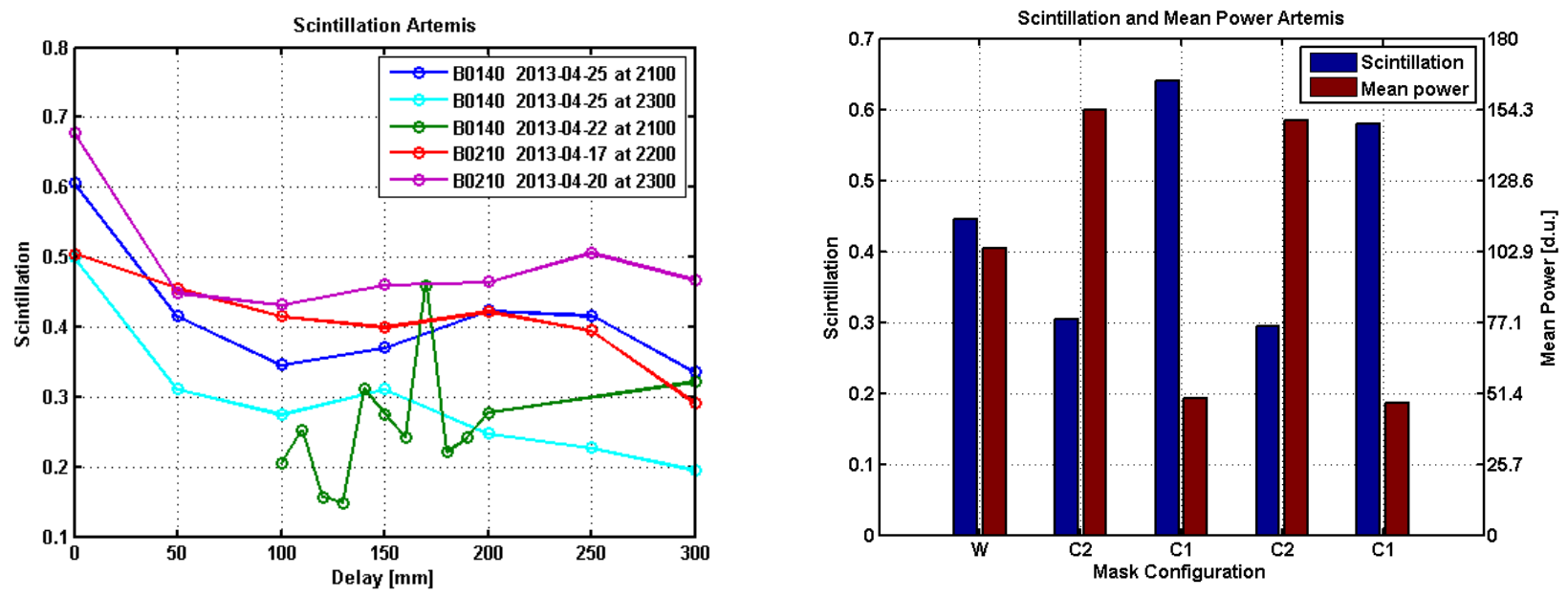

Figure 10. Scintillation versus delay (left) in April 2013 and mask impact on scintillation and mean power (right) for 140 $\mathrm{mm}$ beam width in October 2012.

Inspecting Figure 10 (right), lower scintillation values and higher mean power values are obtained using a mask correlated with the uplink beam. The uncorrelated mask increases drastically the scintillation and decreases the mean power on ARTEMIS. Tracking with the whole aperture represents an intermediate situation between the last two ones. The same behavior was observed during the April measurement campaign, using beam diameters $140 \mathrm{~mm}$. For $210 \mathrm{~mm}$ we observed the same trend but the improvement was much smaller than in the other two configurations.

These measurements used a mask larger than the uplink beam diameter due to power limitations in the TS. The received power from ARTEMIS is quite limited, which does not allow masks in front of the tracking sensor below an apertureequivalent diameter of $100 \mathrm{~mm}$. With such an aperture, the TS power measurements are limited by the quantization error. Therefore, we used the maximum aperture diameter in all cases, to make sure that the link was not lost.

\section{CONCLUSIONS}

During October 2012 and April 2013, two measurement campaigns were carried out with ARTEMIS satellite and the SILEX testbench. At the time of this measurement campaign the ARTEMIS satellite hosted the only optical terminal on a GEO.

During these measurement campaigns, the following parameters were observed: Fried parameter, uplink fading duration and frequency, uplink and downlink scintillation, tracking error, and angle-of-arrival fluctuations. Furthermore, the OGS receive aperture diameter was changed to proof the tracking performance with smaller areas.

Copyright 2014 Society of Photo-Optical Instrumentation Engineers. This paper was published in SPIE Photonics West 2014 and is made available as an electronic reprint with permission of SPIE. One print or electronic copy may be made for personal use only. Systematic reproduction and distribution, duplication of any material in this paper for a fee or for commercial purposes, or modification of the content of the paper are prohibited. 
In this paper, we presented the results of the uplink fading analysis. The fading in the uplink channel is a combination of scintillation and pointing error: the last one due to the lack of correlation between uplink and downlink. The point-ahead angle is larger than the isoplanatic angle and therefore by tracking the downlink the beam wander cannot be fully compensated. The remaining beam wander impact changes with the beam divergence, since larger divergences covers a wider area at the satellite side and therefore fading can be better avoided. In fact, increasing the beam divergence is a simple solution in order to reduce beam wander impact, but with the drawback of reduced available received power at the satellite.

Through these measurements we achieved an experimental proof that transmitter diversity reduces the depth and length of the fades, i.e. in general the fractional fade time. Results were presented at both beam divergences and compared with a single uplink beam. Furthermore, it was shown that tracking on ground with an aperture mask designed to coincide the uplink beam position and size is more powerful than using the whole aperture as long received power of the tracking sensor is high enough.

We would like to thank people of Redu Space Services and IAC, specially Ángel Alonso and Icíar Montilla for their great involvement in the measurement campaigns and Álex Oscoz for his helpfulness, providing valuable meteorological data.

\section{REFERENCES}

[1] Gütlich, B., Meyer, R., Philipp-May, S. and Pagels-Kerp, A., "German roadmap on optical communication in space," Advanced Solid-State Lasers Congress, LM1B.2., OSA (2013).

[2] Fletcher, G. D., Hicks, T. R. and Laurent, B., "The SILEX optical interorbit link experiment," Electronics \& Communication Engineering Journal 3(6), 273-279 (1991).

[3] Tolker-Nielsen, T. and Oppenhauser, G., "In-orbit test result of an operational optical intersatellite link between ARTEMIS and SPOT4, SILEX," Proc. SPIE 4635, 1-15 (2002).

[4] Alonso, A., Reyes, M. and Sodnik,Z., "Performance of satellite-to-ground communications link between ARTEMIS and the optical ground station," Proc. SPIE 5572, 372-383 (2004)..

[5] Romba, J., Sodnik, Z., Reyes, M., Alonso, A. and Bird, A. "ESA's bidirectional space-to-ground laser communication experiments," Proc. SPIE 5550, 287-298 (2004).

[6] Sodnik, Z., Furch, B. and Lutz, H. "Optical intersatellite communication," Selected Topics in Quantum Electronics, IEEE, 16(5), 1051-1057 (2010).

[7] Tolker-Nielsen, T. and Guillen, J-C., "SILEX the first European optical communication terminal in orbit," in ESA bulletin 96 (November 1998).

[8] Andrews, L. and Phillips, R., [Laser Beam Propagation through Random Media], SPIE Press, 2nd edition (2005).

[9] Baykal, Y., "Correlation and structure functions of Hermite-sinusoidal-Gaussian laser beams in a turbulent atmosphere," J. Opt. Soc. Am. A. 21(7), 1290-1299 (2004).

[10] Born and Wolf, [Principles of Optics], Cambridge University Press (1999).

[11] Reyes, M., Alonso, A., Chueca, S., IAC, J. J. F., López, P., Comeron, A., Diós, F., Rodriguez, A., and Rubio, J. A., “ARTEMIS laser link - final report,” Tech. Rep., Instituto de Astrofísica de Canarias (2004).

[12] Giggenbach, D., "Optimierung der optischen Freiraumkommunikation durch die turbulente Atmosphäre - Focal Array Receiver," PhD thesis, Universität Der Bundeswehr München (November 2004).

[13] Sasiela, R. J., [Electromagnetic Wave Propagation in Turbulence], SPIE (2007).

[14] Giggenbach, D., "Deriving an estimate for the fried parameter in mobile optical transmission scenarios," Appl. Opt. 50, 222-226 (Jan 2011).

[15] Svelto, O., [Principles of Lasers], Springer (1998).

Copyright 2014 Society of Photo-Optical Instrumentation Engineers. This paper was published in SPIE Photonics West 2014 and is made available as an electronic reprint with permission of SPIE. One print or electronic copy may be made for personal use only. Systematic reproduction and distribution, duplication of any material in this paper for a fee or for commercial purposes, or modification of the content of the paper are prohibited. 